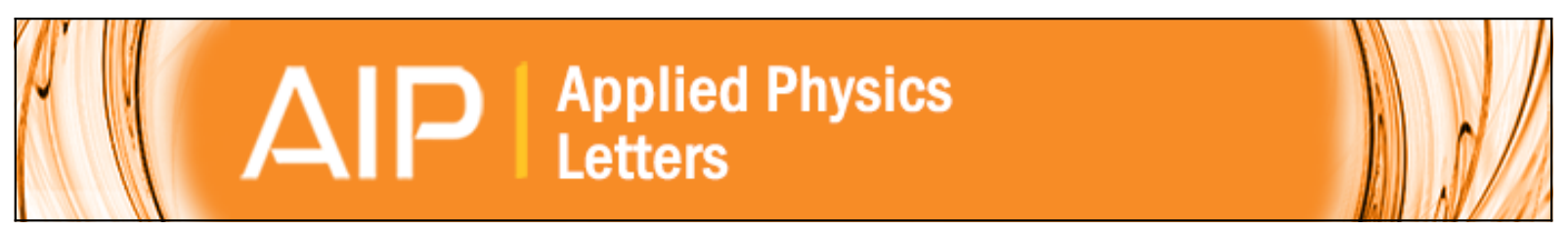

\title{
Ferroelectric-semiconductor photovoltaics: Non-PN junction solar cells
}

Fude Liu, Wentao Wang, Lei Wang, and Guandong Yang

Citation: Applied Physics Letters 104, 103907 (2014); doi: 10.1063/1.4868304

View online: http://dx.doi.org/10.1063/1.4868304

View Table of Contents: http://scitation.aip.org/content/aip/journal/apl/104/10?ver=pdfcov

Published by the AIP Publishing

\section{Articles you may be interested in}

Molecular ferroelectric contributions to anomalous hysteresis in hybrid perovskite solar cells

APL Mat. 2, 081506 (2014); 10.1063/1.4890246

Polymer-based parallel tandem solar cells with a transparent ferroelectric interconnecting layer

Appl. Phys. Lett. 104, 083302 (2014); 10.1063/1.4866398

Interfacial polymer ferroelectric dipole induced electric field effect on the photovoltaic performance of organic solar cells

J. Vac. Sci. Technol. B 31, 04D112 (2013); 10.1116/1.4813752

Origin of the efficiency enhancement in ferroelectric functionalized organic solar cells

Appl. Phys. Lett. 98, 183301 (2011); 10.1063/1.3587630

Study of Lead Free Ferroelectric Films for New Solar Cells

AIP Conf. Proc. 1169, 55 (2009); 10.1063/1.3243271

High-Voltage Amplifiers

- Voltage Range from $\pm 50 \mathrm{~V}$ to $\pm 60 \mathrm{kV}$

- Current to $25 \mathrm{~A}$

Electrostatic Voltmeters

- Contacting \& Non-contacting

- Sensitive to $1 \mathrm{mV}$

- Measure to $20 \mathrm{kV}$
ENABLING RESEARCH AND

INNOVATION IN DIELECTRICS,

ELECTROSTATICS, MATERIALS, PLASMAS AND PIEZOS

Rek www.trekinc.com 


\title{
Ferroelectric-semiconductor photovoltaics: Non-PN junction solar cells
}

\author{
Fude Liu, ${ }^{\text {a) }}$ Wentao Wang, Lei Wang, and Guandong Yang \\ Department of Mechanical Engineering, The University of Hong Kong, Hong Kong, Hong Kong
}

(Received 4 February 2014; accepted 27 February 2014; published online 14 March 2014)

\begin{abstract}
Traditional positive-negative (PN) junction based solar cells have many limitations. Herein, we introduce ferroelectric-semiconductor solar cells that use the bound surface charges of the ferroelectric for achieving charge separation in the semiconductor. The feasibility of the new concept cells was verified both experimentally and theoretically in detail. The new cells are unique in that free charge carriers and fixed charge carriers are physically separated from each other. The feature allows us to go beyond traditional junction-based structures and have more freedom in material selection, device design, and fabrication. (C) 2014 AIP Publishing LLC. [http://dx.doi.org/10.1063/1.4868304]
\end{abstract}

Photovoltaic devices have been traditionally developed for optimizing three key steps for charge carriers: generation, separation, and transport. ${ }^{1,2}$ In particular, how to realize charge carrier separation usually dictates the basic device structure. Traditional solar cells are essentially positivenegative (PN) junction (or $e^{-}$donor $/ e^{-}$acceptor junction) based, and utilize the internal electric field near the junction interface for realizing charge carrier separation. ${ }^{3}$ However, the junction-based structure is just one way to realize the functions of solar cells. In addition, this kind of structure limits material choices and device fabrication to form a workable junction due to issues such as lattice mismatch, doping, and band alignment. Moreover, inevitable heavy doping processes in traditional solar cells result in strong Auger recombination. ${ }^{4}$ In order to overcome the issues that occur with PN junctions, ferroelectric photovoltaic devices have been developed. The typical structure is a ferroelectric layer sandwiched between the top and bottom electrodes. ${ }^{5}$ The device utilizes the ferroelectric layer for both light absorption and charge separation with its internal electric field due to polarization. Because ferroelectric materials in general have large band gaps, short circuit current $\left(\mathrm{I}_{\mathrm{sc}}\right)$, and energy conversion efficiency are very low under sunlight, although high open circuit voltage $\left(\mathrm{V}_{\mathrm{oc}}\right)$ can be obtained. ${ }^{6}$ Another approach is to add low bandgap materials into the ferroelectric matrix (or vice versa) to enhance the light absorption. ${ }^{7}$ The drawback of this approach is that more trapping states are introduced into the device as well. Regan et $a l^{8}{ }^{8}$ showed screening-engineered field-effect solar cells that use the electric field effect as in a field-effect transistor to tune the electrode-semiconductor junctions. However, this approach is challenging for practical applications, because an external bias has to be carefully applied to the gate electrode in order to produce workable field-induced semiconductor PN junctions. It has been shown recently ${ }^{9}$ that a large, permanent, internal electric field can be achieved for enhancing charge separation by incorporating a ferroelectric polymer layer into organic solar cells, which eliminates the need for an external bias. One issue with this approach is that the ferroelectric layer has to be made extremely thin (a few monolayers) for charge carriers to tunnel through it, which

\footnotetext{
${ }^{\text {a) }}$ Author to whom correspondence should be addressed. Electronic mail: fordliu@hku.hk.
}

make it very challenging to fabricate large-area solar cells. Therefore, it is worth exploring other possibilities.

Herein, we introduce a new type of photovoltaic cells that realize the charge carrier separation in the semiconductor with the bound surface charges of the ferroelectric. The new photovoltaic cells, like their traditional counterparts, still use a semiconductor material with suitable energy bandgap and excellent carrier transport as the lightabsorbing layer. In addition, a ferroelectric material is introduced to provide an external electric field on the semiconductor layer for realizing the charge carrier separation therein. Unlike the case in traditional ferroelectric solar cells though, the ferroelectric layer herein is not used for light absorption. Therefore, the new cells can overcome the aforementioned problems with traditional photovoltaic cells, and they are thus promising in providing more flexibilities and possibilities in photovoltaics.

In one embodiment of the new concept (Fig. 1(a)), a solar cell consists of a ferroelectric layer, a semiconductor layer, a pair of poling electrodes ( $P$-electrodes), and a pair of working electrodes (anode and cathode). The polingelectrode patterns are aligned well with those of anode and cathode, respectively. The semiconductor serves the purpose of light absorption. By applying opposite electric fields between the first and second pairs of poling and working electrodes, respectively, corresponding regions in the ferroelectric layer are polarized in opposite directions normal to the horizontal plane. When the ferroelectric is polarized as that shown in Fig. 1(a), it is defined as forward polarization; if the polarization directions are reversed, it is called reverse polarization. After polarization, external electric fields are removed, and then, remnant polarizations are left behind. Anode and cathode thus collect free electrons and holes, respectively, in the semiconductor layer under the influence of the external field due to ferroelectric polarizations; electrons then flow through the external circuit to do work on the load (R) and finally recombine with holes at the cathode. In practice, we prepared a series of cells according to Fig. 1(a) (see Fig. 1(b)). The semiconductor is a p-type single-crystalline Si wafer with thickness of $200 \mu \mathrm{m}$. Ti and Au electrodes (served as the anode and cathode, respectively) were deposited on the $\mathrm{Si}$ wafer with sputtering. The ferroelectric is a $500 \mathrm{~nm}$-thick $\mathrm{BaTiO}_{3}$ layer that was spin coated on the wafer following a normal procedure. ${ }^{10}$ Two $\mathrm{Ag}$ poling electrodes 
were deposited with thermal evaporation. Finally, the cells were wired out with $\mathrm{Ag}$ paste and $\mathrm{Au}$ wire, and then, the entire surface of the ferroelectric was covered by epoxy and glass for insulating. The ferroelectric was poled by following the standard procedure in the literature. ${ }^{11}$ The effective area for each cell is $\sim 78 \mathrm{~mm}^{2}$. The device performance was evaluated with a standard solar simulator (ABET Sun 2000 coupled with Keithley 2602A).

The device performance for a typical cell is shown in Fig. 2. Under dark and before polarization (blue curve in Fig. 2(a)), the $I-V$ curve is a straight line with high resistivity. However, after forward polarization, the I-V curve (red one) shows rectifying behavior as in a PN junction diode. The rectification ratio, defined as the ratio of the positive current over the negative current at $\mathrm{V}= \pm 0.5 \mathrm{~V}$, respectively, is 222 . In addition, the leakage current after forward polarization is greatly reduced compared to the case of reverse polarization that shows in general Ohmic behavior (not shown here). We further investigated the forward-polarization cells under sunlight illumination (Fig. 2(b)). For comparison, the device performance before polarization and under illumination is also included (blue curve in Fig. 2(b)). From the zoom-in view in the inset, there is no photovoltaic output for the cell before polarization. After forward polarization, however, the $\mathrm{I}-\mathrm{V}$ curve shifts downward, exhibiting the photovoltaic effect with $\mathrm{V}_{\mathrm{oc}}=170.5 \mathrm{mV}$ and $\mathrm{I}_{\mathrm{sc}}=0.034 \mu \mathrm{A}$. We also studied the optical response of the cell after forward polarization; a photocurrent was constantly generated whenever the illumination was switched on, and there was no photocurrent whenever the illumination was off. We repeated the experiment with many samples (even with those whose contacts are made of the same material), and the results turned out to be consistent and repeatable. It can then be concluded that free charge carriers in Si can be separated and collected for electricity generation, although the energy conversion efficiency is low at the moment.

(a)

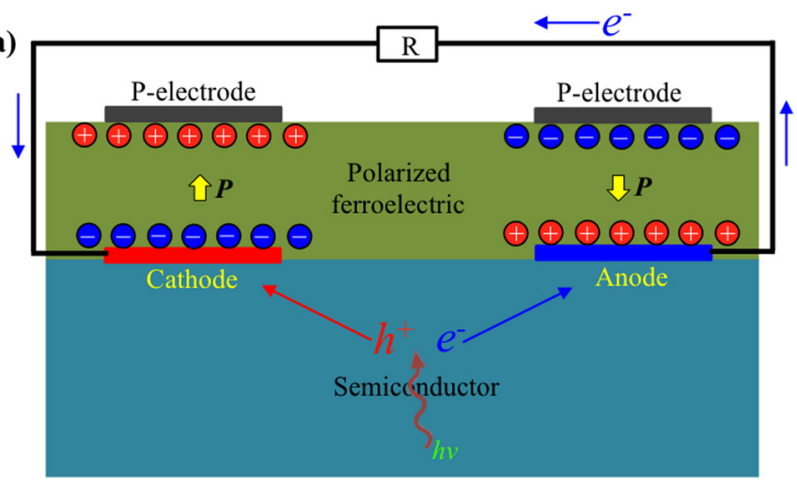

(b)

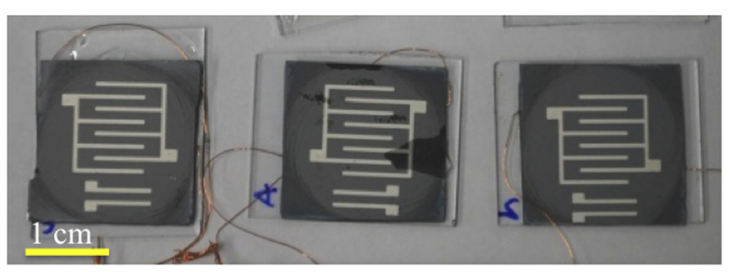

FIG. 1. Ferroelectric-semiconductor photovoltaics. (a) Schematic of the device structure with forward polarization (not drawn to scale). (b) Real solar cells (viewed from the $\mathrm{BaTiO}_{3}$ side).
The poor device performance may be closely related to the following reasons. One main reason is due to metal contacts. On one hand, the metal contact quality with the semiconductor is poor with high resistance as can be seen in Fig. 2. On the other hand, the metal screening effect may weaken the induced electric field in the semiconductor. ${ }^{12}$ Transparent conducting oxides (TCO) with less carrier density and longer screening length may be used as electrodes instead. Because the silicon wafer is too thick $(200 \mu \mathrm{m})$ and sunlight is shot from the $\mathrm{Si}$ side, it may not be easy for charge carriers to be collected effectively by electrodes. Another reason may be related to the possible carrier injection from the semiconductor into the ferroelectric. We know that the energy gaps (work function) of $\mathrm{BaTiO}_{3}$ and $\mathrm{Si}$ are $3.2(5.2)$ and $1.12(4.6) \mathrm{eV}$, respectively, ${ }^{13}$ and the conduction band offset between $\mathrm{BaTiO}_{3}$ and $\mathrm{Si}$ is negative, i.e., $-0.1 \mathrm{eV}$. Therefore, electrons may flow from $\mathrm{Si}$ into $\mathrm{BaTiO}_{3}$, resulting in conduction through the ferroelectric. A thin insulating layer may be added between $\mathrm{Si}$ and $\mathrm{BaTiO}_{3}$ to prevent the charge injection. In addition, there may be other issues such as surface passivation, device layout, and counter-charges at the poling electrodes. All the aforementioned reasons mean huge room for further improving the performance of the new concept cells. At least now we can check in principle the device feasibility by considering the
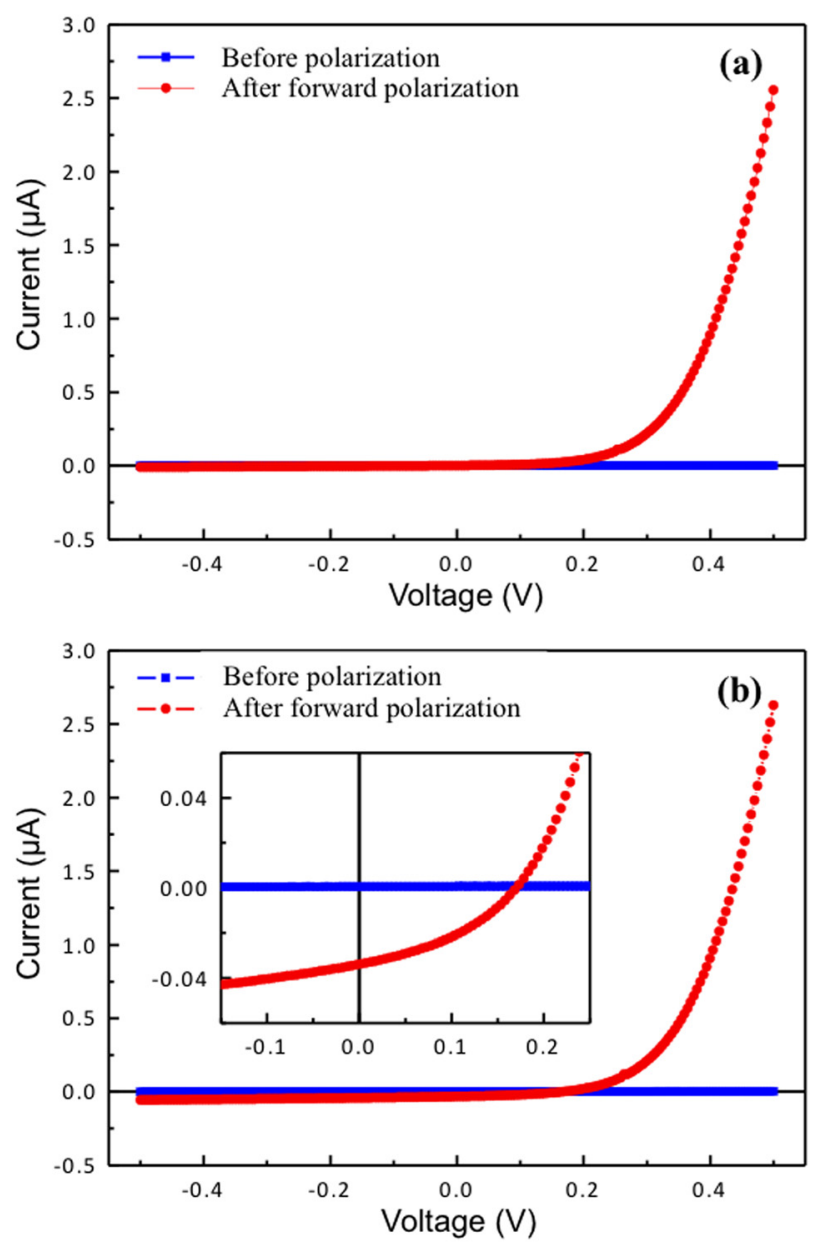

FIG. 2. Device performance of a typical cell. (b) I-V curves of beforepolarization and after-forward-polarization states, respectively, under dark condition. (c) I-V curves of before-polarization and after-forward-polarization states, respectively, under illumination condition. Inset is the zoom-in view. 

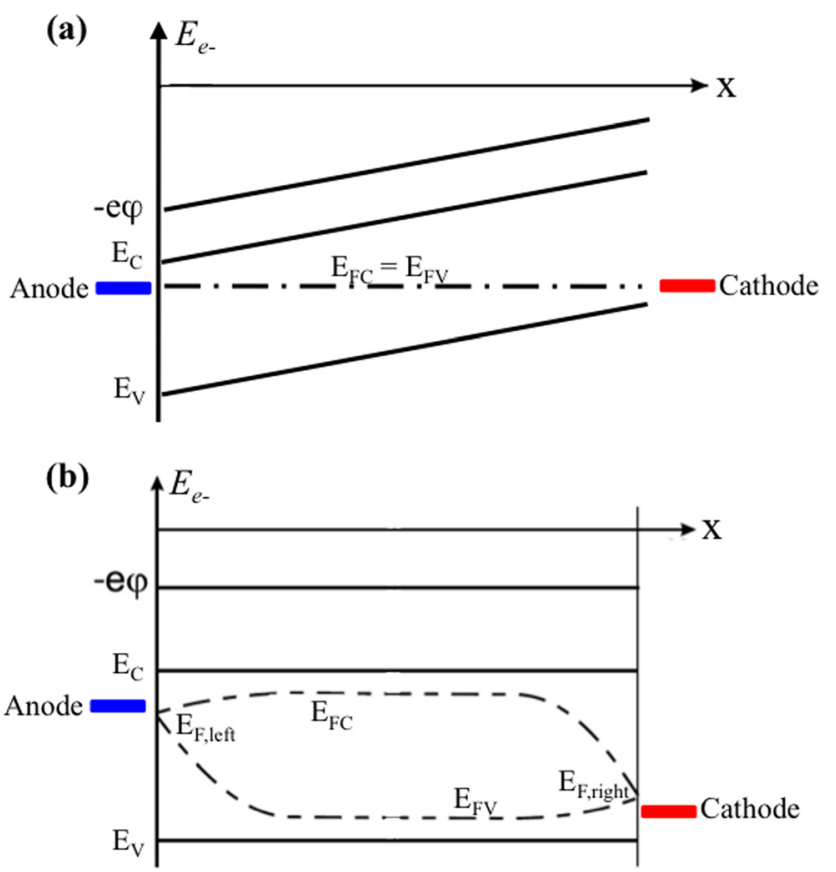

FIG. 3. Effect of an external electric field on the band diagram of a semiconductor (intrinsic). (a) Under dark condition. (b) Under sunlight illumination.

induced electric field in the semiconductor due to the ferroelectric bound surface charges.

First of all, it is important to know the influence of an external electric field (or voltage) on the band diagram of a semiconductor (which is intrinsic in this case) (Fig. 3). Under dark condition (Fig. 3(a)), the external electric field will tilt the semiconductor band diagram, and then electrons and holes will accumulate to the anode and cathode, respectively, until the Fermi level lines up to reach the equilibrium state. If we shoot sunlight on the semiconductor under open-circuit condition (Fig. 3(b)), the Fermi levels in the semiconductor split and more free carriers are accumulated on the electrodes; differences in both Fermi energies (i.e., $V_{o c}=E_{F, \text { left }}-E_{F, \text { right }}$ ) and carrier concentrations occur between two surfaces with strong surface recombination. ${ }^{2}$ Overall, we see that the band diagram shown here is similar to that of a PN solar cell (see also Fig. 4(c)). ${ }^{14}$ Therefore, it is expected that the new solar cells in principle work. Obviously, the feasibility is closely related to the bound surface charges, device layout, and others.

From the simplified version of the new concept cell (Fig. 4(a)), we can calculate the induced electrostatic potential difference $\left(V_{E S}\right)$ due to the ferroelectric polarization $\boldsymbol{P}$. The induced bound surface charges are then given by the following expression:

$$
Q_{P}=A P,
$$

where $A$ is the electrode area. Appearance of surface charges leads to a $V_{E S}$ between the two surfaces of the dielectric. If $C$ is the capacitance, then the $V_{E S}$ can be calculated as

$$
V E S=\frac{Q_{P}}{C}=\frac{A P}{\frac{\varepsilon_{0} \varepsilon_{r} A}{d}}=\frac{d P}{\varepsilon_{0} \varepsilon_{r}},
$$

where $d$ is the spacing between cathode and anode, $\varepsilon_{0}$ the vacuum permittivity $\left(=8.85 \times 10^{-12} \mathrm{~F} / \mathrm{m}\right)$, and $\varepsilon_{r}$ the dielectric constant of the ferroelectric. We can get $V_{E S}=3.9 \mathrm{~V}$ and the corresponding depolarization field $E_{d e p}=V_{E S} / \mathrm{d}=3.9$ $\times 10^{5} \mathrm{~V} / \mathrm{cm}$ in the ferroelectric, when $d=100 \mathrm{~nm}$, $P=0.10 \mathrm{C} / \mathrm{m}^{2}$, and $\varepsilon_{r}=290$ (typical values for ferroelectric films). ${ }^{15}$ Alternatively, we can get the same result with Gauss's law. ${ }^{16}$ Note that $V_{E S}$ is adjustable (see Eq. (2)), for example, by changing the thickness $d$ of the ferroelectric. The spacing between electrodes can be changed as well. It is thus possible for $V_{E S}$ to induce sufficient electric field in the semiconductor. In comparison, for a typical Si PN junction, the fixed ion charge density is $\sim 10^{-4} \mathrm{C} / \mathrm{m}^{2}$, the potential change across the depletion region $\sim 0.85 \mathrm{~V}$, and the maximum value of the electric field at the depletion region $\sim 10^{4} \mathrm{~V} / \mathrm{cm}^{17}$ Therefore, it is convenient to attain suitable electric fields for charge separation in the new cells by choosing right parameters.

Finally, it is worth comparing the new concept cells with traditional PN (or PIN) junction based solar cells. In a simple PN junction solar cell as schematically shown in Fig. 4(b), electrons and holes are separated and transported toward the $\mathrm{n}$ - and $\mathrm{p}$-region, respectively. Only carriers generated in the depletion region and within the diffusion length near the depletion region can be utilized for energy conversion, as indicated by the dashed lines $\left(L_{e-}\right.$ and $L_{h+}$ are the diffusion length of electrons and holes, respectively). By introducing an intrinsic (i) layer between two heavily doped layers $\left(\mathrm{n}^{+}-\right.$and $\mathrm{p}^{+}$-layer), a wider depletion region spanning literally the entire PIN cell (which is essentially still PN junction based) can be achieved (Fig. 4(c)). Overall, in all three cells shown in Fig. 4, photon-generated electrons and holes in the semiconductor are physically separated from each other because of the asymmetric distribution of fixed negative and positive charges. However, the fixed charges (i.e., positive donor ions and negative acceptor ions) in traditional PN (or PIN) junctions are due to ionization of donors and acceptors in the semiconductor and are located therein; in contrast, those (i.e., bound surface charges) in the new cells are caused by the ferroelectric polarization and are
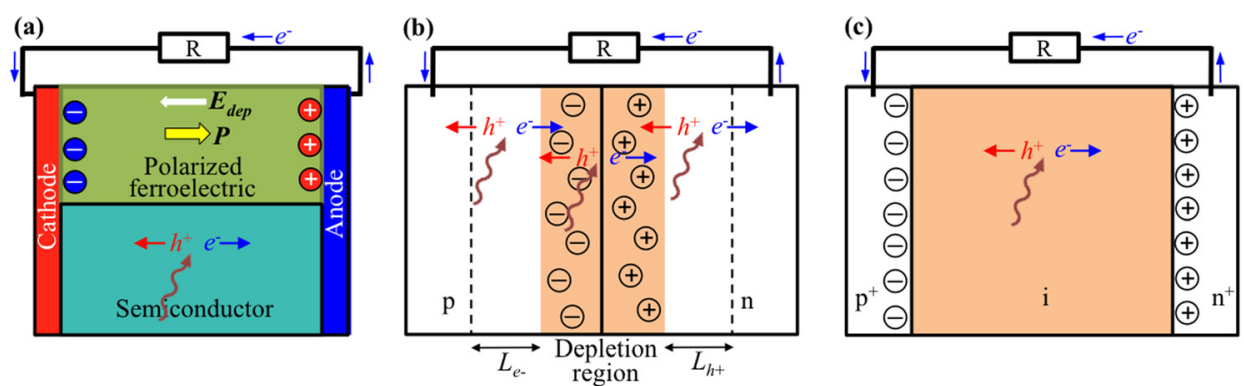

FIG. 4. Comparison between the new concept cell and traditional PN (or PIN) junction based solar cells. (a) Simplified version of the new concept cell. (b) PN junction solar cell. (c) PIN solar cell.

Negative bound surface charge; $\oplus$ Positive bound surface charge; $\ominus$ Fixed acceptor ion; $\oplus$ Fixed donor ion 
located in the ferroelectric instead. Indeed, it is the physical separation between free charge carriers and fixed charge carriers in the new concept that allows us to go beyond traditional junction-based structures, and to have more freedom in material selection, device design, and fabrication in photovoltaics.

In summary, we have demonstrated a new type of photovoltaic cells that realize the charge carrier separation in the semiconductor with the bound surface charges of the ferroelectric. The feasibility of the new cells was verified both experimentally and theoretically in detail. The photovoltaic effect was experimentally observed on the cells with forward polarization only. In addition, it was shown in principle that suitable induced electric fields could be conveniently attained for charge separation in the new cells. In general, the new cells work in a similar fashion as traditional PN (or PIN) junction based counterparts. However, because of the unique physical separation between free charge carriers and fixed charge carriers in the new cells, we can go beyond traditional junction-based structures and have more freedom in material selection, device design, and fabrication in photovoltaics.

The authors acknowledge the financial support from the Seed Funding Programme for Basic Research at HKU (Project Code No. 201211159091), University Development Fund (UDF) No. 2009-2010 (Second Round), GRF Incentive Award No. 2013-2014, Platform Research Projects (Project Code ITP/013/12NP), and HKU Initiative on Clean Energy \& Environment (HKU-ICEE).

${ }^{1}$ Handbook of Photovoltaic Science and Engineering, 2nd ed., edited by A. Luque and S. Hegedus (Wiley, 2010), pp. 61-111; M. A. Green, Third Generation Photovoltaics: Advanced Solar Energy Conversion (Springer, 2005), p. 35; J. Nelson, The Physics of Solar Cells (Imperial College Press, 2003), pp. 17-39.
${ }^{2}$ P. Würfel, Physics of Solar Cells: From Basic Principles to Advanced Concepts, 2nd ed. (Wiley-VCH, 2009), p. 114.

${ }^{3}$ F. Liu, W. Wang, L. Wang, and G. Yang, Nanomater. Energy 2, 3 (2012); M. A. Green, J. Zhao, A. Wang, P. J. Reece, and M. Gal, Nature 412, 805 (2001); I. Gordon, D. Van Gestel, K. Van Nieuwenhuysen, L. Carnel, G. Beaucarne, and J. Poortmans, Thin Solid Films 487, 113 (2005); B. O'Regan and M. Gratzel, Nature 353, 737 (1991); P. C. Choubey, A. Oudhia, and R. Dewangan, Recent Res. Sci. Technol. 4, 99 (2012); M. A. Green, K. Emery, Y. Hishikawa, and W. Warta, Prog. Photovoltaics: Res. Appl. 19, 84 (2011); S. Gunes and N. S. Sariciftci, Inorg. Chim. Acta 361, 581 (2008).

${ }^{4}$ A. Richtera, F. Wernerb, A. Cuevas, J. Schmidt, and S. W. Glunz, Energy Procedia 27, 88 (2012); S. Armstrong, Nat. Photonics 6, 800 (2012).

${ }^{5}$ M. Qin, K. Yao, and Y. Liang, Appl. Phys. Lett. 93, 122904 (2008); D. Cao, C. Wang, F. Zheng, W. Dong, L. Fang, and M. Shen, Nano Lett. 12, 2803 (2012).

${ }^{6}$ S. Y. Yang, J. Seidel, S. J. Byrnes, P. Shafer, C.-H. Yang, M. D. Rossell, P. Yu, Y.-H. Chu, J. F. Scott III, J. W. Ager, L. W. Martin, and R. Ramesh, Nat. Nanotechnol. 5, 143 (2010); K. Yao, B. K. Gan, M. Chen, and S. Shannigrahi, Appl. Phys. Lett. 87, 212906 (2005).

${ }^{7}$ D. Shvydka and V. G. Karpov, Appl. Phys. Lett. 92, 053507 (2008); X. Yang, X. Su, M. Shen, F. Zheng, Yu. Xin, L. Zhang, M. Hua, Y. Chen, and V. G. Harris, Adv. Mater. 24, 1202 (2012).

${ }^{8}$ W. Regan, S. Byrnes, W. Gannett, O. Ergen, O. Vazquez-Mena, F. Wang, and A. Zettl, Nano Lett. 12, 4300 (2012).

${ }^{9}$ Y. Yuan, T. J. Reece, P. Sharma, S. Poddar, S. Ducharme, A. Gruverman, Y. Yang, and J. Huang, Nature Mater. 10, 296 (2011).

${ }^{10}$ H. Kozuka, S. Takenaka, H. Tokita, and M. Okubayashi, J. Eur. Ceram. Soc. 24, 1585 (2004).

${ }^{11}$ K. S. Hwang, J. E. Song, J. W. Jo, H. S. Yang, Y. J. Park, J. L. Ong, and H. R. Rawls, J. Mater. Sci.: Mater. Med. 13, 133 (2002).

${ }^{12}$ M. Ye. Zhuravlev, R. F. Sabirianov, S. S. Jaswal, and E. Y. Tsymbal, Phys. Rev. Lett. 94, 246802 (2005).

${ }^{13}$ L. Hao, Q. Xue, X. Gao, Q. Li, Q. Zheng, and K. Yan, Appl. Phys. Lett. 91, 212105 (2007).

${ }^{14}$ M. Fox, Optical Properties of Solids, 2nd ed. (Oxford University Press, 2010), pp. 363-365.

${ }^{15}$ M. Dawber, K. M. Rabe, and J. F. Scott, Rev. Mod. Phys. 77, 1083 (2005).

${ }^{16}$ D. J. Griffiths, Introduction to Electrodynamics, 3rd ed. (Addison Wesley, 1998), pp. 68-69.

${ }^{17}$ B. Streetman and S. Banerjee, Solid State Electronic Devices (Prentice Hall, 2000), pp. 167-169. 